\title{
Beta through the prism of wavelets
}

\author{
Aasif Shah $^{1^{*}}$ (D, Arif Tali ${ }^{2}$ and Qaiser Farooq ${ }^{2}$
}

\author{
* Correspondence: \\ aasif127@gmail.com \\ ${ }^{1}$ Indian Institute of Technology \\ Madras, Chennai, India \\ Full list of author information is \\ available at the end of the article
}

\begin{abstract}
In this paper, we empirically show how wavelet decomposition can provide an easy vehicle to study the systematic risk properties of return series to serve as protocol for different traders who view the market with different time resolutions. By using the separate catalogue of Large Cap, Mid Cap and Small Cap stocks comprising S\&P BSE500 index of Indian capital market, we report that the conventional beta coefficients estimated from CAPM are essentially an average of wavelet betas but the later provides a resolution more appropriate and hence need to be considered in a realistic risk assessment of securities. Additionally, the wavelet beta coefficients for Large Cap stocks are found more stable than Mid and Small capitalized stocks. This paper is the first attempt of its kind to link the underlying methodology across different capitalized stocks to identify the precise beta in a complex market behavior.

Keywords: CAPM, Beta, Wavelet

JEL classification: C22, C49, G21
\end{abstract}

\section{Introduction}

Multi-scale representations are more effective in characterising the time-frequency properties of financial return series. It is rather more practical and reliable in view of the large number of investors who participate in the stock market and take decisions over different time periods. Basically, stock market participants are a diverse group comprising intraday traders, hedging strategists, portfolio managers, financial and non-financial institutions and so on. It is notable that these market participants operate on different time scales depending upon their requirements and thus the true dynamic structure of the relationship between variables might vary over different time scales. The purpose of this paper is to address one of such issues related to Capital Asset Pricing Model which makes its inferences on efficient markets and believes that economic agents are entities that act according to the rational expectation strategy. The model builds on Markowitz $(1952,1959)$ mean variance portfolio theory and conveys the notion that securities are priced such that the expected returns will compensate investors for the expected risks. The CAPM presumes investors to be utility maximising agents and allows predicting the return of an asset for its given level of systematic risk measured through beta coefficient. However, very often evidence showed the inability of the CAPM to identify the true beta that would allow investors to price risky securities in order to determine the desirability of an investment. One of

(C) The Author(s). 2018 Open Access This article is distributed under the terms of the Creative Commons Attribution 4.0 International License (http://creativecommons.org/licenses/by/4.0/), which permits unrestricted use, distribution, and reproduction in any medium, provided you give appropriate credit to the original author(s) and the source, provide a link to the Creative Commons license, and indicate if changes were made. 
the reasons of the incapability of the CAPM is because the model is based on the assumptions which appear to be unrealistic in real market world. For instance, one of such assumptions is about investor homogeneity. The main characteristic of this paradigm is the representative agent, i.e., the investors are homogeneous about their preferences, their expectations and their investment strategies. However, the differences in planning horizons, frequency of trading or institutional constraints are neglected. While Fama (1965) and Levhari and Levy (1977) profoundly criticized the homogenous assumption of the model, the work of M. Levy and Levy (1996) showed that homogeneous expectation assumption could lead to a highly inefficient market with periodic and predictable booms and crashes. Long-back, the veteran economist Keynes (1930) also argued that agents do not have sufficient knowledge of the structure of the economy to form correct mathematical expectations that would be held by all agents. Of late Muller et al. (1997) emphasized that the formation of markets is based on multiple layers of investment horizons or time scales ranging from seconds to years and hence market participants cannot be a single homogeneous group of investors. Thus, under the homogenous expectations assumption, the CAPM model fails to recognize diverse kinds of traders seeking investment betas for different time horizons. For instance, there are traders who take a very long view for the investment and consequently concentrate on what is termed as 'market fundamentals'. These traders ignore ephemeral phenomena. In contrast, other traders trade on a much shorter time-scale and as such are interested in temporary deviations of the market. And yet other traders may operate in the market for which even a day is long time. Each of these classes of traders analyses their own trading with their own perception of yardstick, consistent with their trading horizons. Miller (1977) proposes a direct relationship between a stocks risk and its divergence of opinion. He argues that "in practice, uncertainty, divergence of opinion about a security's return, and risk go together". Consequently, he proposes that "the riskiest stocks are also those about which there is the greatest divergence of opinion". An early empirical study by Bart and Masse (1981) supports Miller's proposition.

Therefore, if CAPM homogeneity assumption must be true, then all investors are supposed to experience the same degree of systematic risk measured by its 'beta' for their investments. But this is not the case as CAPM was criticized mainly based on time varying beta. However, such conclusions were direct reflections of time domain analysis where the evolution of individual variables were examined and assessed over time. This was apparent because traditionally it was impossible to look simultaneously at the same dynamic system through time and frequency domains. In other words, the time and frequency domains were not interchangeable, i.e., information was bound to be lost in changing return series from one domain to another. To deal with such issues, the adaptations of econometric techniques in financial analysis were not able to visualize financial markets as complicated dynamic systems to address the heterogeneous behavior of investors who operate at different time and frequency resolutions. For example, researchers while addressing the interval effect of beta use conventional time decomposition scales like daily, weekly, monthly or annually. However, under such circumstances, when the return interval is changed from daily to weekly and so on, the number of sample points would decrease which obviously amounts in the loss of information. This was one of the fundamental reasons as why physicists have been lured into financial markets to bring 
new theories to study the chaos of the markets and to model the price of complex derivatives without losing information in terms of data. The example of losing sample points in the conventional data decomposition is illustrated diagrammatically later in this paper. Wavelets possess many desirable properties, some of which are useful in economics and finance. It can enable the finance or economics researcher to separate out a return series into its constituent multi-resolution components to discover the complex market behavior. Additionally, wavelets can deal with both stationary and non-stationary data. Thus, the main purpose of the paper is to repeat the conventional beta time varying analysis in simultaneous time frequency oscillations by using the insights from wavelets to improve the specification and estimation of asset pricing models that incorporate this risk dynamics. The remainder of the paper proceeds as follows. Review Literature section provides an insight of the related empirical literature. Multiscale Model Specification section discusses about the methodological aspect in terms of wavelets. Data Description and Empirical Results section demonstrates the data and findings of the study. We conclude in the final section.

\section{Review literature}

Investments in the capital market exposes investors to market risk and hence analyzing such risks are critical part of investing. Recognizing the importance of market risk, Sharpe (1964) developed the CAPM as an extension of Markowitz's modern portfolio theory Markowitz 1959. According to CAPM, beta is the only relevant measure of a stocks risk that explains how investors should act and price risky securities. Though CAPM is considered as one of the investment pillar on which the entire development of financial theory is based but it is also true that CAPM has been the most debatable asset pricing model because of its rigid assumptions and poor empirical record. The model has a long history of theoretical and empirical investigations of market beta estimations. Amongst many others, some studies of beta estimations concentrated on the borrowing constraints Black (1972); non-synchronous data issues Scholes and Williams (1977); the time horizon of investors Levhari and Levy (1977); stability of beta over time Harvey (1989); the impact of return interval Handa et al. (1989); the effect of world markets and volatility Bekaert \& Harvey (1997) and so on. Specifically, the studies on the impact of return interval on beta estimates point out the importance of the timescale issue. Although there is a consensus about time variation in market betas, but it is not clear how this variation can possibly be captured. One of the reasons of the incapability of the CAPM is because the model is based on the assumptions which appear to be unrealistic in real market world. One of such assumptions is investor homogeneity. The main characteristic of this paradigm is the representative agent, i.e., the investors are homogeneous about their preferences, their expectations and their investment strategies. However long back Keynes (1930) argued that agents do not have sufficient knowledge of the structure of the economy to form correct mathematical expectations that would be held by all agents. Also, the heterogeneous market hypothesis of Muller et al. (1997) emphasizes that the formation of markets is based on multiple layers of investment horizons or time scales ranging from seconds to years and hence market 
participants cannot be a single homogeneous group of investors. Hence if CAPM homogeneity assumption must be true, then all investors are supposed to experience the same degree of systematic risk measured by its 'beta' for their investments. An early study during 1970's by Blume (1971) shows that individual betas are unstable, and the instability declines as the length of observation decreases. His results were however confirmed by Baesel (1974). Several years later, Levhari and Levy (1977) also argued that a deviation from true horizon can cause a systematic bias in the regression coefficient. Also, Gordon and Norman (1980) stated that one of the fundamental reasons for beta instability is measurement error, that is, theoretical beta relates ex-ante expectations while estimated beta relates ex-post observations. Whereas Scott and Brown (1980) argued that this type of measurement error combined with auto correlation in the residuals would result in unstable estimates. Similarly, Fama and French (2004) believed many strange assumptions like beta coefficient is constant through time and all investors have the same single investment horizon are responsible to the empirical invalidity of the CAPM model. Several papers have proposed extensions of the CAPM to allow incorporation of time-variation. One of the most widely used method to estimate beta as a time series process is the Kalman (1960). It has been applied for the estimation of betas and tests for beta constancy in several markets including the Indian market by Moonis and Shah (2003). The Kalman Filter allows beta to be estimated as a time-varying stochastic process. During the past two decades, the time varying nature of beta has been reported time and again both in developed as well as developing markets and the Indian market is not an exception. However, the results drawn by earlier studies could not be treated as conclusive as they failed to capture the beta variations in simultaneous time frequency oscillations. The fact is that while market consists of heterogeneous market participants (such as investors, speculators, financial institutions and so on) the economic theories make its inferences on efficient markets based on a model in which economic agents are entities who act according to the rational expectation strategies. With these presumptions, econometric tools were not adequate to characterize and break down the complex heterogonous market behavior. This was first dealt by Gençay et al. (2005) who applied wavelet decomposition by using data sets from US, UK and Germany. They came up with an interesting conclusion that market provides a stronger relationship between portfolio return and risk as the wavelet scale increases from higher to lower frequencies of data. Hiroshi (2005) also examined multi-scale beta estimation approach based on wavelet analysis for Japanese stock between 1983 and 03 using Discrete Wavelet Transform (DWT) analysis and concluded that the conventional beta estimates although are useful in most cases but wavelet-based beta estimates are useful for understanding the sensitivity of the returns of securities to the returns on the market index in a much deeper sense. In another attempt, Viviana Fernandez (2006) supported CAPM at a medium-term horizon for the data of Santiago stock exchange between 1997 and 02 . He also concluded that risk is concentrated at higher frequencies of the data with VaR model across wavelet scales. Of late, Rua and Nunes (2012) attempted to measure market risk for 23 developed and 23 emerging markets between 1998 and 08 using continuous wavelet transform 
analysis. Their results showed that beta coefficient was relatively stable at lower frequencies presenting a value of around 1 . In contrast, at higher frequencies, the beta coefficient varied considerably attaining values as high as 3 in some economic episodes. Deo and Shah (2012) were the first to incorporate wavelets in beta estimation for Indian capital market. They calculated two betas based on the short periodicity and long periodicity of market returns based on (MODWT). Their findings revealed that conventional beta estimate is an "average" of the wavelet-based beta estimates for most of the cases.

\section{Multiscale model specification}

Before describing wavelets as an alternative refinement tool to improve the statistical inference of the data in a potentially revealing manner, a diagrammatic representation of the effect of different time intervals on beta estimation based on conventional time series decomposition is given in Fig. 1.

As it is evident from the figure that the estimated beta of the stock decreased from 0.62 (daily) to 0.39 (quarterly) with increased time intervals. This example shows that it makes a difference if one employs daily, weekly or monthly data to estimate systematic

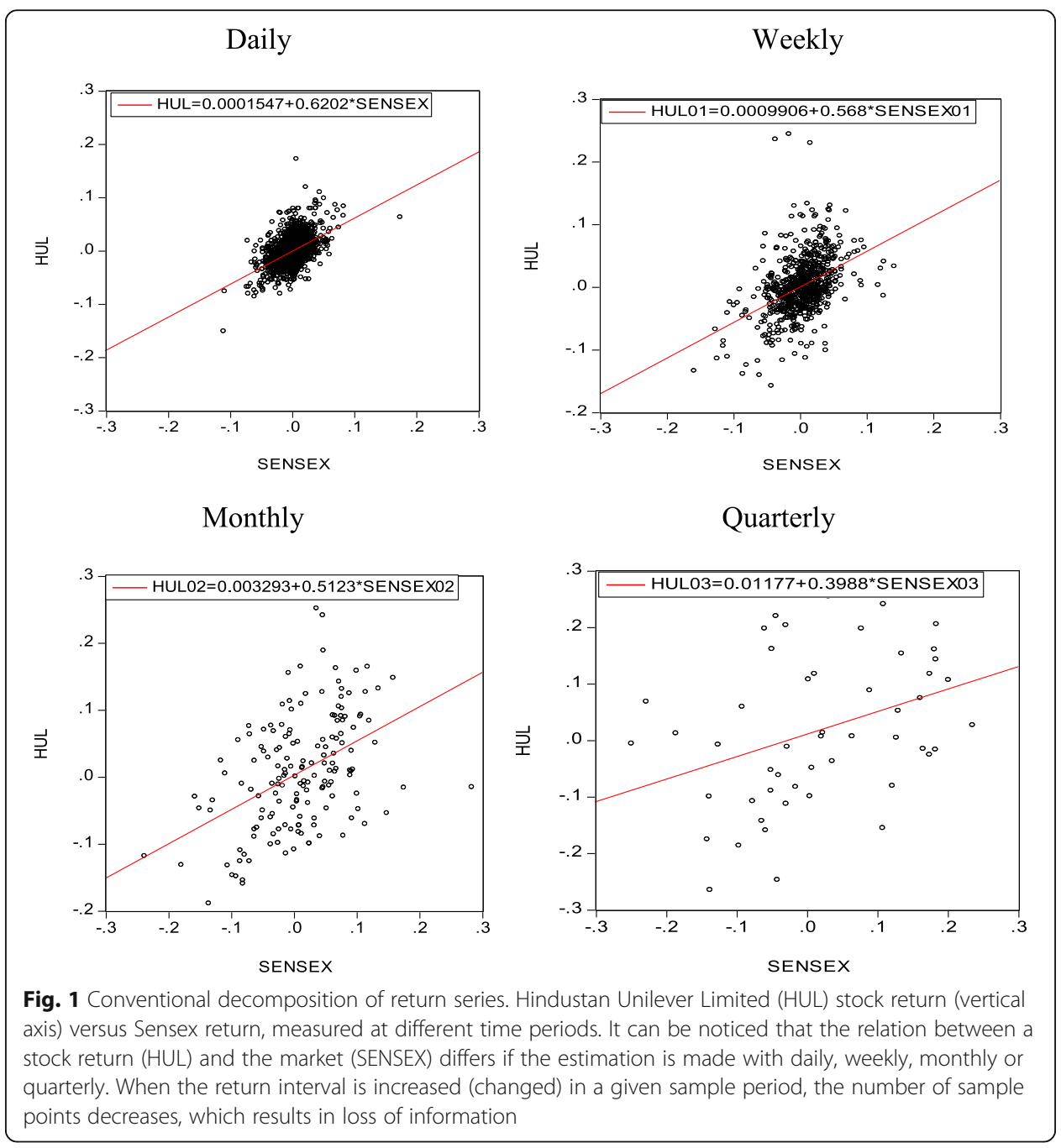


risk. However, it can be noticed that when the return interval is increased in a given sample period, the number of sample points decreases, from 2750 (daily data), to 550 (weekly data) to 132 (monthly data) to 44 (quarterly data) which results in loss of time information. Therefore, it is crucial to look beyond the conventional decomposition of financial data to preserve the time information irrespective of the number of decompositions or frequency intervals. Wavelet analysis is relatively new in economics and finance, although the literature on wavelets is growing rapidly. This method has started to find place in economic and financial applications in recent years Oral and Gazanfar (2017a, 2017b). The term wavelets literally mean small waves, as they have finite length (compactly supported) and oscillatory behaviour. They are types of basic functions that are used to decompose a function $f(t)$, i.e. a signal, a surface, a series, etc., in more elementary functions which include information about $f(t)$. Wavelet analysis is a refinement of Fourier analysis. The problem with the Fourier transform is that it loses the time information as it looks deep into the frequency values of the series and therefore it cannot be used successfully to study the nonstationary financial time series data Oral and Gazanfar (2017a, 2017b). Though the Short-Windowed Fourier Transform (SWFT) emerged to overcome the time frequency concern but still (SWFT) could not solve the resolution problem. However, of late, discrete wavelet transform emerged as an easy vehicle to study the multi-scale properties of a process both in terms of time and frequency. There are two kinds of wavelets: mother wavelets $\psi(t)$ and farther wavelets $\phi(t)$.

$$
\int \psi(t) d t=0, \int \phi(t) d t=1
$$

The former effectively represent the detail and high-frequency parts of time series, while the latter are good in representing the smooth and low-frequency components. Unfortunately, except some special case, there is no analytical formula for computing a wavelet function. Wavelets are usually derived using special two-scale dilation equation. For a father wavelet $\phi(t)$ a dilation equation has the following view

$$
\phi(t)=\sqrt{2} \sum_{k} l_{k} \phi(2 t-k)
$$

A mother wavelet $\psi(t)$ is related to a father by

$$
\psi(t)=\sqrt{2} \sum_{k} h_{k} \psi(2 t-k)
$$

The coefficients $l_{k}$ and $h_{k}$ are defined as

$$
l_{k}=\frac{1}{\sqrt{2}} \int \phi(t) \phi(2 t-k) d t, h_{k}=\frac{1}{\sqrt{2}} \int \psi(t) \phi(2 t-k) d t .
$$

They are essentially the low-pass and high-pass filter coefficients. As it often happens in practical problems, it is the time series (sequence of values) that is dealt with rather than continuous function defined over real axis. In this case short sequences of values called wavelet filters are employed and denoted by $\left\{h_{k}\right\}_{k=0}^{L}, \mathrm{~L}$ is the number of values in the sequence. The filter coefficients $h_{k}$ however has the following restrictions: 


$$
\sum_{k=0}^{L-1} h_{k}=0, \quad \sum_{k=0}^{L-1} h_{k}^{2}=1, \quad \sum_{k=0}^{L-1} h_{k} h_{k+2 j}=0 \quad(\mathrm{j} \text { is any non-zero integer })
$$

Coefficients $l_{k}$ and $h_{k}$ from (1.4) are related through the following expression $l_{k}$ $=(-1)^{k+1} h_{L-1-k}, k=0,1, \ldots, L-1$.

The problem associated with the application of the DWT for time series analysis is that it suffers from a lack of translation invariance. This means that circularly shifting a time series will not necessarily shift its DWT coefficients in an equivalent manner. This problem can be tackled by means of a highly redundant non-orthogonal transform called the maximal overlap discrete wavelet transform $(\mathrm{MODWT})^{1}$ that is shift-invariant in the sense that circular shifts in the time series results in the same circular shift in the MODWT coefficients. This is not true of the DWT. In the DWT, a circular shift in the time series can cause changes in the distribution of signal energy across scales. This is often undesirable. The fact that the MODWT has the same number of coefficients at each scale as the original data means that the MODWT has the same time resolution as the data at each scale. In many applications, it is critical to be able to associate changes in the wavelet transform coefficients with times in the original data. This is much easier to do with the MODWT than with the DWT where the time resolution is decreasing as the scale increases. Moreover, the critically sampled discrete wavelet transform (DWT) is defined only for signals which are a power of two in length. The MODWT retains many of the nice properties of the DWT such as energy preservation and variance decomposition while removing the restriction to have a length which is a power of two. Therefore, the analysis of the present work has been carried out with MODWT and Daubechies least asymmetric (LA) wavelet filter of length $\mathrm{L}=8$ based on eight non-zero coefficients with periodic boundary conditions. The MODWT is applied up to level $\mathrm{J}=7$ that produces one vector of smooth coefficients i.e. $\mathrm{s} 7$ representing the smooth behavior of the data at the coarse scale and seven vectors of details coefficients $d 1, d 2, d 3$, $\mathrm{d} 4, \mathrm{~d} 5, \mathrm{~d} 6$ and $\mathrm{d} 7$ representing progressively finer scale deviations from the smooth behavior. Through the synthesis or reconstruction operation, the original signal can be re-assembled from the wavelet and scaling coefficients using the inverse stationary wavelet transform. Specifically, with $J=7$, It is possible to deconstruct seven wavelet details vectors and one wavelet smooth vector each associated with a time scale $2^{\mathrm{j}-1}$. Since daily data to estimate beta coefficients is used, the first level $\mathrm{d} 1$ captures market behavior for two days period, $\mathrm{d} 2$ for 4 days period, D3 for eight days period, D4 for 16 days period, D5 for 32 days period, D6 for 64 days period, D7 for 128 days period and S7 captures longer period respectively. The smooth and detail components obtained from the deconstruction process take the form of non-periodic oscillating waves representing the long-term trend and the deviations from it at an increasing level of detail.

Consider a return series $\mathrm{X}$ with arbitrary sample size $\mathrm{N}$, the $j_{t h}$ level MODWT wavelet $\left(\tilde{W}_{J}\right)$ and scaling $\left(\tilde{V}_{J}\right)$ coefficients are defined as,

$$
\tilde{W}_{J, T} \equiv \sum_{l=0}^{L_{j-1}} \tilde{h}_{j, l} X_{t-1 \bmod N} \tilde{V}_{J, T} \equiv \sum_{l=0}^{L_{j-1}} \tilde{g}_{j, l} X_{t-1 \bmod N}
$$

Where $\tilde{h}_{j, l} \equiv h_{j, l} / 2^{j / 2}$ are the MODWT wavelet filters, and $\tilde{g}_{j, l} \equiv g_{j, l} / 2^{j / 2}$ are the MODWT scaling filters. For a stock return series $X$ with $N$ samples, the MODWT yields an additive decomposition or MRA given by 


$$
X=\sum_{J=1}^{J_{0}} \tilde{D}_{J}+\tilde{S}_{J 0}
$$

Where $\tilde{D}_{j, t}=\sum_{l=0}^{N-1} \tilde{h}_{j, l} \tilde{W}_{j, t+l \bmod N} \& \tilde{S}_{j, t}=\sum_{l=0}^{N-1} \tilde{g}_{j, l} \tilde{V}_{j, t+l \bmod N}$.

According to Eq. (7), a set of coefficients $\left\{D_{J}\right\}$ are obtained with the same number of samples $(\mathrm{N})$ as in the original return series $(\mathrm{X})$. These are called wavelet "details" and they capture local fluctuations over the whole period of a return series at each scale. The set of values $S_{J 0}$ provide a "smooth" or overall "trend" of the original return series. Adding $D_{J}$ to $S_{J 0}$, for $\mathrm{j}=1,2, \ldots, J_{0}$, gives an increasingly more accurate approximation of the original signal. This additive form of reconstruction allows predicting each wavelet sub-series separately and adding the individual predictions to generate an aggregate forecast. The wavelet coefficients are usually arranged in collection from coarse scales to finest.

$$
W=\left(\begin{array}{c}
\vec{S}_{J} \\
\vec{d}_{J} \\
\vec{d}_{J-1} \\
\dddot{\vec{d}}_{1}
\end{array}\right)
$$

Where, $\vec{S}_{J}=\left(S_{J, 1} ; S_{J, 2} ; \ldots ; S_{J, \frac{n}{J}}\right), \vec{d}_{J}=\left(d_{J, 1} ; d_{J, 2} ; \ldots ; d_{J, \frac{n}{J}}\right), \vec{d}_{J-1}=\left(d_{J-1,1} ; d_{J-1,2} ; \ldots ;\right.$ $\left.d_{J-1, \frac{n}{2-1}}\right), \vec{d}_{1}=\left(d_{1,1} ; d_{1,2} ; \ldots d_{1, \frac{n}{2}}\right)$. Each of vectors $\vec{s}_{J}, \vec{d}_{J}, \vec{d}_{J-1}, \ldots, \vec{d}_{1}$ is called a crystal. More detail descriptions of crystals can be found in Ramsey ${ }^{2}$ (2002). The Eq. (8) present decomposition of time series into time-frequency oscillations. Figure 2 shows the deconstruction of the return series into frequency components through pyramid algorithm.

If $x(t)$ represents an original stock return series say (daily) then $w_{1}(t)$ denotes first level wavelet decomposition scale that captures $(1 \sim 2)$ days stock return fluctuation in the market and $v_{1}(t)$ denotes smooth scale. Further to capture (2-4) days stock return fluctuation, smooth series, that is, $\left(v_{1}(t)\right)$ require decomposition which in turn will produce $w_{2}(t) \& v_{2}(t)$. Here $v_{2}(t)$ denotes smooth series for further decomposition and $w_{2}(t)$ is associated up to eight days stock return fluctuations. Figure 3 shows details and smooth crystals of BSE-S\&P 500 index returns. As the wavelet scale is increasing, the wavelet coefficients become thicker and hence analyzing such patterns assume an integral part of an investment analysis.

Shrinkage methods are used on the MODWT object, the output of imodwt may differ from the original series. After de-construction of excess returns $R_{i t}-R_{f t} \& R_{m t}-R_{f t}$, following equation is estimated at each scale (details as well as smooth).

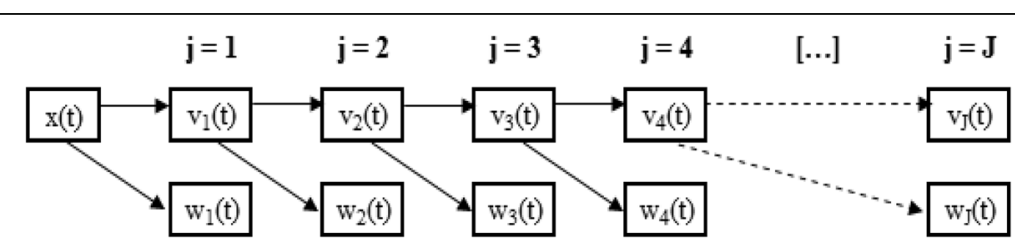

Fig. 2 Flowchart of the pyramid algorithm 


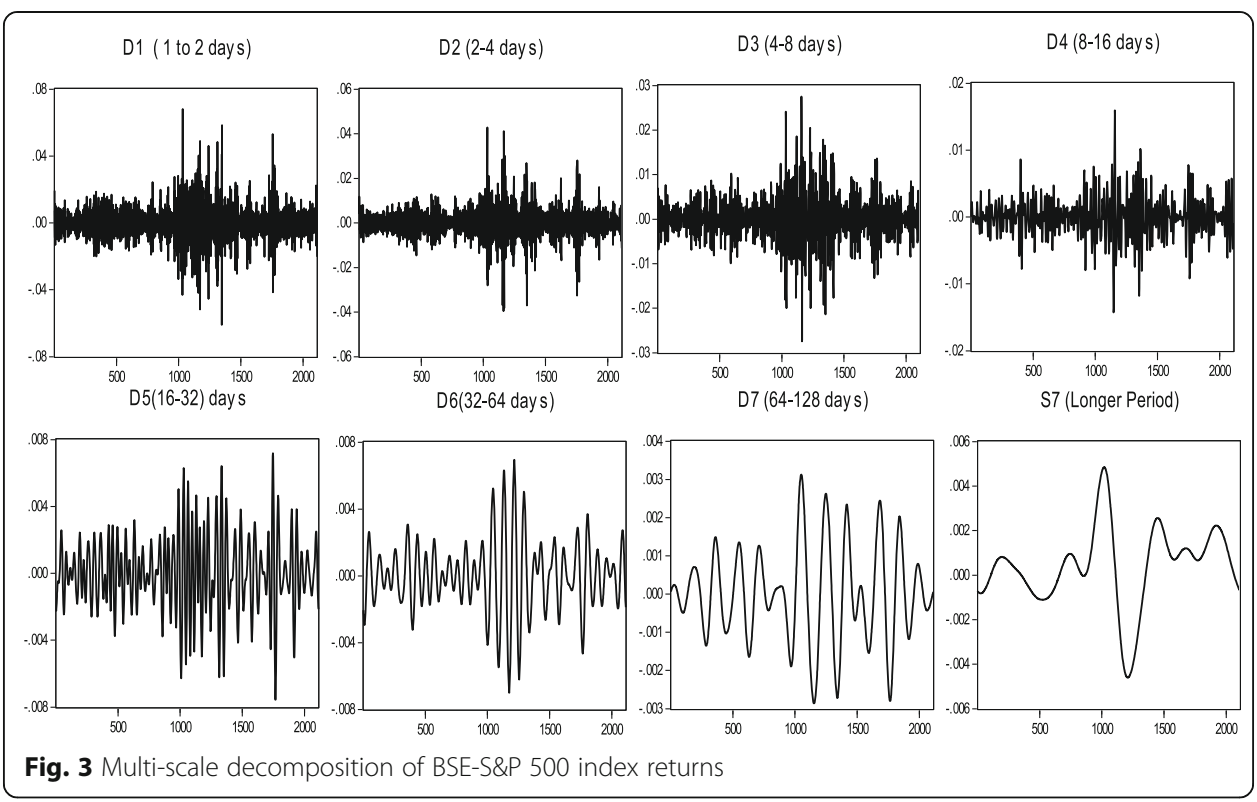

$$
E\left(r_{i t}\right)=\alpha_{i}+\beta\left[E\left(r_{m}\right)+\varepsilon_{i}\right]
$$

To have a basic understanding of the wavelet phenomenon, it is imperative to return to the early example of HUL with wavelet MODWT decomposition ranging from high frequency (D1) to low frequency (S3). Figure 4 shows beta slope at different wavelet scales. It can be noticed that when the return interval is increased in a given sample period, the number of sample points remains the same and hence no loss of information. The practical implementation of the MODWT requires handling boundary conditions. The most natural method for dealing with the boundary is to assume that the length $N$ series is periodic, and to grab observations from the other end to finish the computations. Another way to handle the boundary is to impose the brick wall condition which prohibits convolutions that extend beyond the ends of the series. This boundary condition is appropriate in an analysis when there is no compelling reason to assume that the data are periodic and symmetric in structure. Finally, it is imperative to choose the wavelet filter. A reasonable choice of the filter must consider the specific analysis goal that has to be achieved such as isolation of transient events in a time series, analysis of variance and multi-resolution analysis. The final analysis is based on MODWT using LA(8) filter as it could yields coefficients that can be approximately uncorrelated between scales while having a filter width short enough such that the impact of boundary conditions is tolerable.

\section{Data description and empirical results}

The data set consists of all stocks comprising S\&P BSE-500 index between January 01, 2005 and June 30, 2013. ${ }^{3}$ Attempt has been made to segregate the index and to construct a separate catalogue of Large Cap, Mid Cap and Small Cap stocks based on their market capitalisation structure. Additionally, sample stocks have been classified into respective industries. The S\&P BSE 500 index has been taken as the corresponding benchmark for Large Cap, Mid Cap and Small Cap stocks respectively. The sample size 


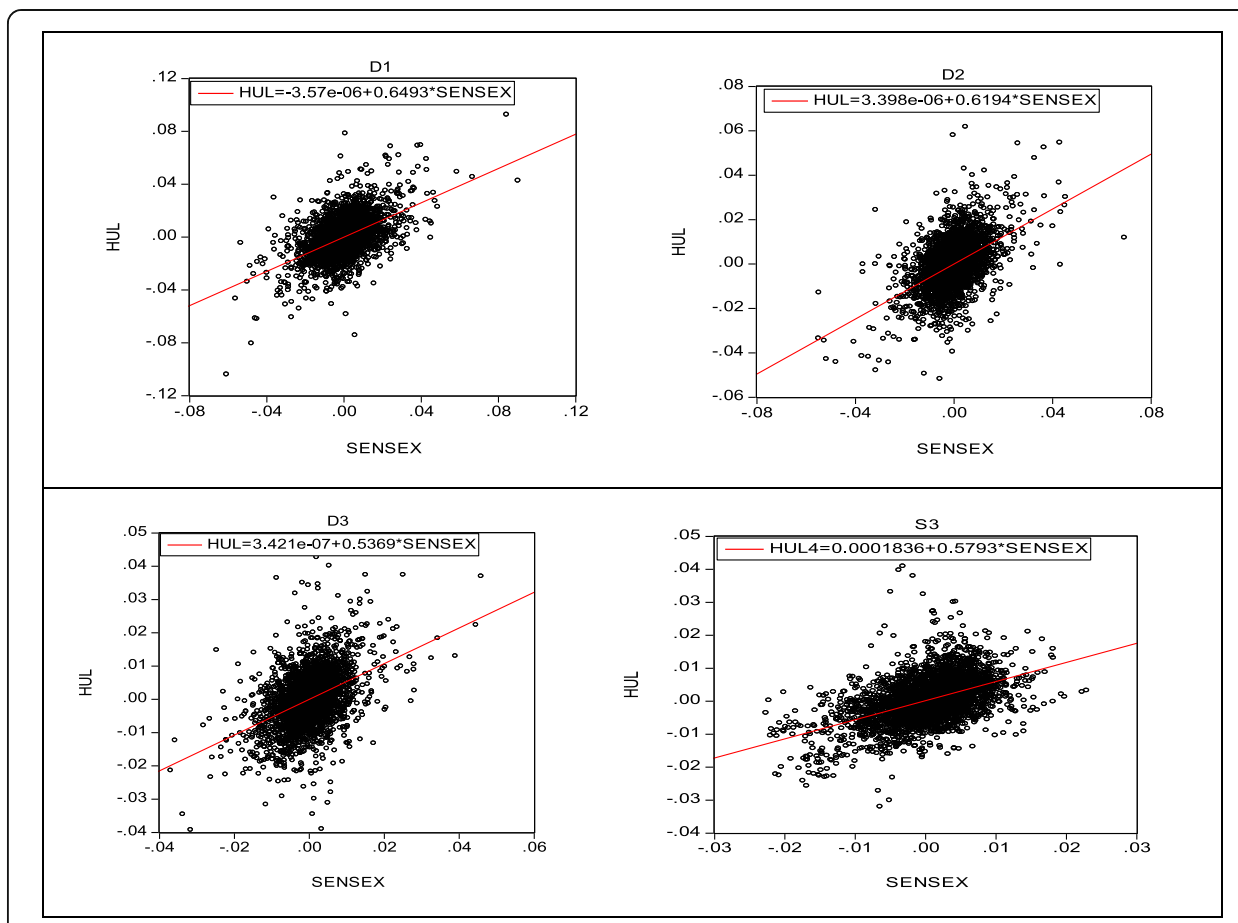

Fig. 4 Wavelet decomposition of return series. Hindustan Unilever Limited stock return (vertical axis) versus Sensex return, measured at different time scales based on wavelet decomposition. The wavelet scales are such that scale one (D1) corresponds to the time period of 1-2 days, scale two (D2) - 2 to 4 days, Scale three (D3)- 4 to 8 and S3 represents the undefined but longer time period. It can be noticed that when the return interval is increased in a given sample period, the number of sample points remains the same and hence no loss of information

is 2112 market days or roughly eight and half years. The daily return of each stock $R_{i t}$ is calculated as the $\log$ price difference i.e., $\log p_{i t}-\log _{p i t-1}$ where $p_{i t}$ is the price of asset $\mathrm{i}$ at day t. The market return $r_{m t}$ is taken as the log difference of the S\&P BSE 500 index i.e., $\log S_{t}-\log S_{t-1}$ where $S_{t}$ is the index value at day t. The risk-free rate of return $r_{t}$ is assumed to be the daily Mumbai Interbank offered rate (MIBOR $)^{4}$ for the sample period. With the above data set, the beta estimation of stocks for each capitalised category is carried out with wavelet methodology using MODWT estimator.

Using Eq. (9), conventional CAPM betas have been estimated first followed by wavelet betas at each scale. This is done by allowing wavelet detail and smooth decomposition to $R_{i t} \& R_{m t}$. The return series of each industrial stock has been decomposed into seven wavelet scales and one smooth scale. Overall 288, decomposed series that is, $36 * 8$ have been generated from 36 return series (10 from Large cap, 13 from Mid Cap and 13 from Small Cap) respectively. Given the utility of the data, wavelets scales are such that Scale 1 i.e., D1 is associated with 2 days market period, Scale $2,3,4,5,6,7$ are associated with $4,8,16,32,64$ and 128 days market period respectively. The final scale (S7) captures the long-term stock as well as market behaviour. The coefficient Beta $(\beta)$ is the key parameter around which the analysis of this paper revolves. If beta found is essentially similar across wavelet scales, $J$ then there is little reason to believe that wavelet betas are useful. However, if the estimated wavelet betas across scales differ significantly, then the return interval chosen arbitrarily would be inappropriate. 
Table 1 summarizes CAPM betas on original and decomposed return series derived from MODWT analysis for large capitalized stocks. The estimation results reveal that the beta coefficient for Capital Goods industry for instance according to CAPM is 0.94 on original or un-decomposed return series. But subsequently after deconstruction of the return series without losing any data points, different beta parameters emerged at different scales. The table reveal that D1 that is associated with 2 days period produced beta coefficient with 0.90, D2 with 0.93, D3 with 0.99, D4 with 0.95, D5 with 1.02, D6 with 0.90 , D7 with 1.24 and finally S7 with 1.109 . The wavelet beta coefficients imply that the risk intensity of the Capital Goods industry stock depends on the time scales. The results indicate that the stock is less volatile during 2, 4, 8, 16 and 64 days investment period but more volatile during 32 and 128 days period. The smooth scale (S7) depicts the higher volatility of stock during long but an undefined period. Overall the results demonstrate that beta becomes stronger as the time scales increases. The results are noteworthy. The evidence of monotonic increase in beta coefficient from (D1 to D2) is evident for 8 industrial stocks with the exceptions of IT and Pharmaceutical stocks. However, the beta for some industrial stocks like Capital Goods, Bank, Oil and Transport increase monotonically from (D1 to D3). The stocks of IT illustrate a different pattern. The beta component for this industry decreases with the increase in time scale. Furthermore, it appears from the table that beta for IT, Oil \& Gas, Pharmaceutical, Transport and FMCG stock remain less volatile across the specified wavelet scales. Whereas, stocks of Metal and Power industry continued to be volatile across underlying scales. Overall the results are more revealing. The beta coefficients for Large Cap stocks on an average tend to move by a smaller percentage against any given movement in the market. The evidence is supported by all wavelet scales. The last two columns tabulate the $F$-values against the null hypothesis that estimated beta coefficients at wavelet scale D1 (that represents two days market behaviour) is different from the wavelet scale (D5) representing one-month market behaviour. It can be noticed that out of ten Large Cap industries, the beta component differs significantly for two industries such as IT and Metal. This implies that the beta coefficients for the rest of the industrial stocks remain stable by and large between these two-time intervals. We also compared beta coefficients at (D5) and beta coefficients at (D7) representing four

Table 1 Wavelet betas for Large Cap stocks

\begin{tabular}{lclllllllllll}
\hline Industry & CAPM Beta & D1 & D2 & D3 & D4 & D5 & D6 & D7 & S7 & Mean (D1 to S7) & D1 = D5 & D1 = D7 \\
\hline CG & $\mathbf{0 . 9 4}$ & 0.91 & 0.94 & 1.00 & 0.96 & 1.02 & 0.90 & 1.25 & 1.11 & $\mathbf{1 . 1 1}$ & 1.51 & $2.82^{\mathrm{a}}$ \\
IT & $\mathbf{0 . 8 3}$ & 0.91 & 0.83 & 0.74 & 0.69 & 0.67 & 0.71 & 0.56 & 0.97 & $\mathbf{0 . 7 7}$ & $1.90^{\mathrm{b}}$ & 0.75 \\
Banking & $\mathbf{1 . 1 3}$ & 1.04 & 1.16 & 1.29 & 1.28 & 0.99 & 1.33 & 1.29 & 0.99 & $\mathbf{1 . 1 6}$ & 0.67 & $2.89^{\mathrm{a}}$ \\
Metal & $\mathbf{1 . 2 2}$ & 1.16 & 1.29 & 1.17 & 1.33 & 1.44 & 1.23 & 1.32 & 1.58 & $\mathbf{1 . 3 2}$ & $2.88^{\mathrm{a}}$ & 1.53 \\
O\&G & $\mathbf{0 . 7 1}$ & 0.69 & 0.73 & 0.75 & 0.62 & 0.91 & 0.80 & 0.60 & 0.59 & $\mathbf{0 . 7 1}$ & 1.18 & 1.18 \\
Pharmacy & $\mathbf{0 . 6 0}$ & 0.60 & 0.56 & 0.65 & 0.60 & 0.69 & 0.71 & 0.82 & 0.61 & $\mathbf{0 . 6 5}$ & 1.10 & 1.03 \\
Housing & $\mathbf{0 . 9 2}$ & 0.89 & 0.93 & 0.83 & 1.12 & 1.10 & 1.06 & 1.01 & 1.15 & $\mathbf{1 . 0 2}$ & 1.69 & 0.57 \\
Power & $\mathbf{1 . 1 0}$ & 1.08 & 1.15 & 1.01 & 1.24 & 1.01 & 1.22 & 1.00 & 1.01 & $\mathbf{1 . 0 9}$ & 0.81 & 0.51 \\
Transport & $\mathbf{0 . 6 8}$ & 0.62 & 0.66 & 0.78 & 0.86 & 0.75 & 0.76 & 0.83 & 0.93 & $\mathbf{0 . 7 8}$ & 0.76 & 0.63 \\
FMCG & $\mathbf{0 . 5 5}$ & 0.51 & 0.59 & 0.54 & 0.62 & 0.66 & 0.55 & 0.56 & 0.59 & $\mathbf{0 . 5 8}$ & 0.91 & 0.56 \\
Overall & $\mathbf{0 . 8 5}$ & 0.81 & 0.86 & 0.89 & 0.93 & 0.90 & 0.93 & 0.94 & 0.91 & $\mathbf{0 . 9 0}$ & 0.63 & 0.49 \\
\hline
\end{tabular}

Superscripted letters ${ }^{\mathrm{a}, \mathrm{b}}$, denote values with statistical significance at $1 \%$, and $5 \%$ respectively CG, O\&G and CD denotes Capital Goods; Oil \& Gas and Consumer Durables Industry respectively 
months' time period. However, we again observed little evidence about the statistical difference between these two-time intervals. The possible reason may be explained by the immovable trading attitude of the Large Cap investors who invest a larger proportion of their corpus based on certain beliefs that Large Cap companies have ability to generate wealth through their strong corporate governance policies.

Table 2 summarizes the corresponding explanatory power $R^{2}$ values for estimated beta coefficients. It is evident that $R^{2}$ increase monotonically from (D1 to D3) with the exceptions of IT and Pharmaceutical stocks. However, when investment horizon is extended for 16 days period i.e., up to (D4) scale, R-square falls slightly for most of stocks except for Housing, Power and Transport. The R-square start increasing again when the investment horizon in extended beyond 16 days but not more than 64 days. This implies that market is less able to explain the stock variations during 16 and beyond 64 days investment horizon.

Table 3 highlights the beta coefficients of 13 Mid Cap industrial stocks. The monotonic increase in beta coefficients is evident from D1 to D2 for 11 industrial stocks with the exceptions of IT and Housing stocks. However, beta increases monotonically from D1to D5 for some stocks like Capital Goods, Diversified, and FMCG stocks. Additionally, the beta for Oil \& Gas, Agriculture and Pharmaceutical stocks indicate that these stocks remained less volatile than the market for all the traders ranging from days to years. However, it is observed that Housing stock among all industrial stocks are subject to big swings in prices than the market at all frequency intervals. Whereas one important point to be observed here is that among all wavelet scales, sixty-four days investment period i.e., D6 moves less in response to movements of overall market. The evidence is apparent for 9 out of 13 industrial stocks. On the other hand, the highest volatility is found to be associated with 32 days investment period i.e., scale D5. The evidence is obvious for 8 out of 13 industrials. However, like Large Cap stocks, the beta coefficient for overall stocks operating in Mid Cap industries also remained less volatile than the market across specified scales. Unlike large cap stocks, the estimated betas values at D1 are different from the wavelet scale at (D2) for five industrial stocks. The difference is more visible with seven industrial stocks when beta values at (D5) are compared with beta values at (D7). This probable reason of significant or instable beta

Table 2 Corresponding wavelet R-squares

\begin{tabular}{|c|c|c|c|c|c|c|c|c|c|}
\hline Industry & CAPM & D1 & D2 & D3 & D4 & D5 & D6 & D7 & S7 \\
\hline Capital Goods & 0.64 & 0.60 & 0.63 & 0.71 & 0.67 & 0.70 & 0.79 & 0.91 & 0.81 \\
\hline IT & 0.50 & 0.53 & 0.47 & 0.53 & 0.42 & 0.39 & 0.63 & 0.50 & 0.59 \\
\hline Banking & 0.76 & 0.64 & 0.78 & 0.86 & 0.82 & 0.79 & 0.87 & 0.78 & 0.86 \\
\hline Metal & 0.69 & 0.63 & 0.70 & 0.71 & 0.72 & 0.85 & 0.84 & 0.73 & 0.87 \\
\hline Oil \& Gas & 0.56 & 0.50 & 0.59 & 0.65 & 0.55 & 0.67 & 0.79 & 0.54 & 0.77 \\
\hline Pharmaceutical & 0.53 & 0.51 & 0.50 & 0.57 & 0.48 & 0.65 & 0.82 & 0.67 & 0.60 \\
\hline Housing & 0.57 & 0.53 & 0.58 & 0.62 & 0.63 & 0.70 & 0.75 & 0.59 & 0.77 \\
\hline Power & 0.64 & 0.60 & 0.64 & 0.69 & 0.71 & 0.76 & 0.86 & 0.80 & 0.66 \\
\hline Transport & 0.60 & 0.51 & 0.60 & 0.75 & 0.75 & 0.74 & 0.84 & 0.77 & 0.80 \\
\hline FMCG & 0.46 & 0.40 & 0.49 & 0.54 & 0.45 & 0.58 & 0.62 & 0.52 & 0.62 \\
\hline Overall & 0.88 & 0.82 & 0.90 & 0.95 & 0.95 & 0.97 & 0.98 & 0.97 & 0.97 \\
\hline
\end{tabular}


Table 3 Wavelet betas for Mid Cap stocks

\begin{tabular}{lclllllllllll}
\hline Industry & CAPM Beta & D1 & D2 & D3 & D4 & D5 & D6 & D7 & S7 & Mean (D1 to S7) & D1 = D5 & D1 = D7 \\
\hline CG & $\mathbf{0 . 9 5}$ & 0.89 & 0.95 & 0.97 & 1.11 & 1.12 & 0.85 & 1.19 & 1.12 & $\mathbf{1 . 0 3}$ & $2.50^{\text {a }}$ & 1.19 \\
IT & $\mathbf{1 . 0 1}$ & 1.04 & 0.92 & 1.03 & 1.04 & 1.06 & 0.98 & 0.88 & 1.14 & $\mathbf{1 . 0 1}$ & 0.80 & $1.87^{\mathrm{b}}$ \\
Banking & $\mathbf{0 . 9 4}$ & 0.86 & 1.01 & 1.00 & 1.06 & 0.92 & 1.01 & 0.81 & 0.95 & $\mathbf{0 . 9 5}$ & 0.69 & 0.87 \\
Metal & $\mathbf{1 . 1 0}$ & 1.04 & 1.28 & 1.06 & 0.89 & 1.19 & 0.68 & 1.45 & 1.44 & $\mathbf{1 . 1 3}$ & $1.88^{\mathrm{b}}$ & $2.56^{\mathrm{a}}$ \\
O\&G & $\mathbf{0 . 8 8}$ & 0.83 & 0.89 & 0.92 & 0.93 & 0.93 & 0.96 & 0.89 & 0.74 & $\mathbf{0 . 8 9}$ & 1.03 & 0.67 \\
Pharmacy & $\mathbf{0 . 6 5}$ & 0.61 & 0.70 & 0.61 & 0.68 & 0.67 & 0.72 & 0.58 & 0.60 & $\mathbf{0 . 6 5}$ & 0.65 & 1.10 \\
Housing & $\mathbf{1 . 1 9}$ & 1.22 & 1.15 & 1.11 & 1.20 & 1.32 & 1.33 & 1.13 & 1.38 & $\mathbf{1 . 2 3}$ & 1.18 & $1.82^{\mathrm{b}}$ \\
Power & $\mathbf{0 . 9 1}$ & 0.44 & 1.06 & 1.14 & 1.25 & 1.11 & 0.98 & 0.71 & 0.90 & $\mathbf{0 . 9 5}$ & $4.20^{\mathrm{a}}$ & $3.15^{\mathrm{a}}$ \\
Trans. & $\mathbf{0 . 8 9}$ & 0.84 & 0.90 & 0.92 & 0.90 & 0.93 & 0.95 & 1.05 & 1.10 & $\mathbf{0 . 9 5}$ & 0.77 & 1.08 \\
Agriculture & $\mathbf{0 . 6 6}$ & 0.63 & 0.73 & 0.55 & 0.77 & 0.70 & 0.75 & 0.59 & 0.67 & $\mathbf{0 . 6 8}$ & 1.51 & 0.81 \\
FMCG & $\mathbf{1 . 0 5}$ & 1.02 & 1.07 & 1.10 & 1.12 & 1.22 & 0.92 & 1.08 & 0.77 & $\mathbf{1 . 0 4}$ & $2.80^{\mathrm{a}}$ & $2.70^{\mathrm{a}}$ \\
CD & $\mathbf{0 . 9 9}$ & 0.94 & 1.00 & 0.96 & 1.19 & 1.08 & 1.12 & 0.71 & 1.15 & $\mathbf{1 . 0 2}$ & 1.38 & $3.83^{\mathrm{a}}$ \\
Diversified & $\mathbf{0 . 7 4}$ & 0.55 & 0.76 & 0.90 & 0.91 & 1.08 & 0.81 & 1.29 & 1.00 & $\mathbf{0 . 9 1}$ & $4.59^{\mathrm{a}}$ & $3.31^{\mathrm{a}}$ \\
Overall & $\mathbf{0 . 9 1}$ & 0.84 & 0.91 & 0.95 & 0.92 & 0.97 & 0.97 & 0.97 & 0.97 & $\mathbf{0 . 8 9}$ & 1.18 & 0.76 \\
\hline
\end{tabular}

Superscripted letters ${ }^{\mathrm{a}, \mathrm{b}}$, denote values with statistical significance at $1 \%$, and $5 \%$ respectively

CG, O\&G and CD denotes Capital Goods; Oil \& Gas and Consumer Durables Industry respectively

coefficients may be explained by the friction in the trading process which could delay the response of securities' prices to new information for instance.

Table 4 presents the corresponding explanatory power $R^{2}$ values of the wavelet betas for Mid Cap stocks. It can be noticed that when investment horizon is extended for 16 days period that is up to D4 scale, $R^{2}$ falls slightly for most of stocks except for O\&G, Pharmaceutical, Housing, FMCG, Consumer Durables and Diversified stocks. The $R^{2}$ start increasing yet again when the investment horizon in extended beyond 16 days but not more than 64 days. This again implies that market is less able to explain the stock variations during 16 and beyond 64 days investment horizon period.

Table 5 report wavelet betas for Small Cap stocks. Unlike Large and Mid-Cap stocks, the evidence of monotonic increase in beta from D1 to D2 is evident with only 6

Table 4 Corresponding wavelet R-squares

\begin{tabular}{|c|c|c|c|c|c|c|c|c|c|}
\hline Industry & CAPM & D1 & D2 & D3 & D4 & D5 & D6 & D7 & S7 \\
\hline$\overline{C G}$ & 0.80 & 0.73 & 0.80 & 0.85 & 0.82 & 0.86 & 0.89 & 0.92 & 0.91 \\
\hline IT & 0.68 & 0.65 & 0.62 & 0.73 & 0.72 & 0.75 & 0.83 & 0.83 & 0.79 \\
\hline Bank & 0.81 & 0.75 & 0.82 & 0.85 & 0.85 & 0.84 & 0.89 & 0.77 & 0.89 \\
\hline Metal & 0.45 & 0.37 & 0.48 & 0.47 & 0.39 & 0.47 & 0.53 & 0.66 & 0.76 \\
\hline$O \& G$ & 0.66 & 0.58 & 0.62 & 0.73 & 0.78 & 0.74 & 0.81 & 0.78 & 0.77 \\
\hline Pharma & 0.69 & 0.65 & 0.69 & 0.71 & 0.72 & 0.73 & 0.85 & 0.73 & 0.62 \\
\hline Housing & 0.78 & 0.76 & 0.76 & 0.79 & 0.79 & 0.84 & 0.92 & 0.80 & 0.82 \\
\hline Power & 0.47 & 0.18 & 0.44 & 0.49 & 0.45 & 0.54 & 0.55 & 0.60 & 0.63 \\
\hline Transport & 0.73 & 0.66 & 0.71 & 0.79 & 0.78 & 0.78 & 0.89 & 0.92 & 0.88 \\
\hline FMCG & 0.65 & 0.57 & 0.65 & 0.68 & 0.74 & 0.72 & 0.85 & 0.74 & 0.72 \\
\hline Agriculture & 0.68 & 0.59 & 0.70 & 0.73 & 0.71 & 0.77 & 0.86 & 0.84 & 0.74 \\
\hline$C D$ & 0.58 & 0.51 & 0.56 & 0.63 & 0.66 & 0.74 & 0.81 & 0.71 & 0.77 \\
\hline Diversified & 0.50 & 0.30 & 0.45 & 0.63 & 0.66 & 0.72 & 0.77 & 0.95 & 0.95 \\
\hline Overall & 0.87 & 0.81 & 0.91 & 0.94 & 0.95 & 0.97 & 0.98 & 0.97 & 0.93 \\
\hline
\end{tabular}


Table 5 Wavelet betas for Small Cap stocks

\begin{tabular}{lclllllllllll}
\hline Industry & CAPM Beta & D1 & D2 & D3 & D4 & D5 & D6 & D7 & S7 & Mean (D1 to S7) & D1 = D5 & D1 = D7 \\
\hline CG & $\mathbf{1 . 0 3}$ & 1.06 & 1.03 & 1.00 & 1.03 & 0.92 & 1.00 & 1.17 & 0.95 & $\mathbf{1 . 0 3}$ & 1.41 & $2.76^{\mathrm{a}}$ \\
IT & $\mathbf{1 . 1 1}$ & 1.06 & 1.03 & 1.02 & 0.96 & 0.87 & 0.95 & 0.81 & 1.02 & $\mathbf{0 . 9 6}$ & $1.92^{\mathrm{b}}$ & 1.03 \\
Bank & $\mathbf{1 . 1 1}$ & 1.08 & 1.12 & 1.08 & 1.23 & 1.04 & 1.15 & 1.26 & 1.18 & $\mathbf{1 . 1 3}$ & 0.68 & $2.83^{\mathrm{a}}$ \\
Metal & $\mathbf{1 . 0 8}$ & 1.12 & 1.04 & 1.07 & 1.07 & 1.06 & 1.08 & 1.13 & 1.22 & $\mathbf{1 . 0 8}$ & 0.58 & $1.76^{\mathrm{b}}$ \\
O\&G & $\mathbf{1 . 1 9}$ & 1.17 & 1.22 & 1.27 & 1.10 & 1.27 & 1.19 & 1.07 & 1.10 & $\mathbf{1 . 1 5}$ & $1.75^{\mathrm{b}}$ & $2.704^{\mathrm{a}}$ \\
Parma & $\mathbf{0 . 8 4}$ & 0.83 & 0.89 & 0.85 & 0.78 & 0.90 & 0.81 & 0.70 & 0.76 & $\mathbf{0 . 8 2}$ & 0.66 & $1.70^{\mathrm{b}}$ \\
Housing & $\mathbf{1 . 1 9}$ & 1.22 & 1.22 & 1.12 & 1.08 & 1.21 & 1.17 & 1.40 & 1.21 & $\mathbf{1 . 2 1}$ & 0.69 & $3.66^{\mathrm{a}}$ \\
Power & $\mathbf{1 . 0 5}$ & 0.98 & 0.96 & 1.10 & 1.15 & 0.99 & 1.55 & 1.20 & 1.21 & $\mathbf{1 . 1 3}$ & 0.81 & $2.806^{\mathrm{a}}$ \\
Transport & $\mathbf{0 . 9 1}$ & 0.90 & 0.94 & 0.88 & 0.95 & 0.95 & 0.82 & 0.89 & 0.85 & $\mathbf{0 . 9 0}$ & 0.76 & 0.83 \\
FMCG & $\mathbf{0 . 5 5}$ & 0.82 & 0.89 & 0.93 & 0.99 & 0.80 & 0.95 & 0.70 & 0.88 & $\mathbf{0 . 8 7}$ & 0.71 & 0.66 \\
Agricultur & $\mathbf{0 . 9 2}$ & 0.98 & 0.91 & 0.87 & 0.78 & 0.95 & 0.85 & 0.92 & 0.77 & $\mathbf{0 . 9 0}$ & 0.63 & 0.63 \\
CD & $\mathbf{0 . 8 5}$ & 0.84 & 0.76 & 0.90 & 0.97 & 0.85 & 0.95 & 0.66 & 1.15 & $\mathbf{0 . 8 5}$ & 0.80 & $1.68^{\mathrm{b}}$ \\
Diversified & $\mathbf{1 . 2 1}$ & 1.20 & 1.23 & 1.21 & 1.18 & 1.19 & 1.39 & 1.15 & 1.14 & $\mathbf{1 . 2 2}$ & 0.66 & 0.86 \\
Overall & $\mathbf{1 . 0 1}$ & 1.11 & 1.02 & 1.01 & 1.01 & 0.98 & 1.03 & 0.99 & 1.10 & $\mathbf{1 . 0 1}$ & 1.13 & 0.63 \\
\hline
\end{tabular}

Superscripted letters ${ }^{\mathrm{a}, \mathrm{b}}$, denote values with statistical significance at $1 \%$, and $5 \%$ respectively

CG, O\&G and CD denotes Capital Goods; Oil \& Gas and Consumer Durable industries respectively

industrial stocks. However, there is only one industrial stock FMCG for which beta increases monotonically from D1 to D4. The table reports the higher price swings for Bank, Metal, Oil \& Gas, and Housing and Diversified stocks across all frequency intervals. By and large, it appears that both short and long-term investors are exposed to same level of risk when holding stocks in these specific industries. On the other hand, there is smaller movement in the stock prices of Pharmaceutical, Transport, FMCG, Agriculture and Consumer durables across all wavelet scales. However, an interesting observation to be noticed is that unlike Large and Mid Cap stocks, the overall prices of Small Cap stocks in general tend to move more than the market across all frequency intervals except for 32 and 128 days investment period i.e., at D5 \& D7. Moreover, the prices of small capitalised stocks swing more than the market in two, four, eight and sixteen day's investment periods. The evidence is apparent for seven industrial stocks. Like Large Cap stocks, there is little evidence of statistical difference between estimated beta values at D1 and beta values at (D2). However, like Mid Cap stocks, the difference is more evident with eight industrial stocks when beta values at (D5) are compared with beta values at (D7).

Table 6 summarizes $R^{2}$ values of wavelet betas for Small Cap stock. Unlike Large and Mid-Cap stocks, where corresponding explanatory falls mostly when investment horizon is extended either for 16 and 128 days investment period, the R-square for Small-Cap stocks started falling only when the investment horizon is extended for 128 days investment period. This implies that market in general is more able to explain the stock variations for 64 days investment horizon period. It is noteworthy to observe here that for Capital Goods, Agricultural, IT and Housing stocks, beta coefficients generally decreased from D1 to D4 with the increase in wavelet scale. The decrease in beta value for IT stock is evident also from Large Cap sample.

A more interesting result appears from observing the behavior of $R^{2}$ for all stocks viz., Large, Mid and Small-Cap from D6-D7 which decreased when investment horizon is about 128 days period or roughly four months. This means that systematic risk of 
Table 6 Corresponding wavelet R-squares

\begin{tabular}{|c|c|c|c|c|c|c|c|c|c|}
\hline Industry & CAPM & D1 & D2 & D3 & D4 & D5 & D6 & D7 & S7 \\
\hline CG & 0.83 & 0.79 & 0.81 & 0.86 & 0.87 & 0.88 & 0.92 & 0.89 & 0.91 \\
\hline IT & 0.72 & 0.67 & 0.70 & 0.77 & 0.76 & 0.77 & 0.84 & 0.75 & 0.84 \\
\hline Bank & 0.64 & 0.55 & 0.61 & 0.71 & 0.76 & 0.73 & 0.85 & 0.88 & 0.89 \\
\hline Metal & 0.78 & 0.73 & 0.76 & 0.80 & 0.80 & 0.86 & 0.90 & 0.86 & 0.93 \\
\hline O\&G & 0.67 & 0.57 & 0.65 & 0.76 & 0.76 & 0.81 & 0.86 & 0.74 & 0.74 \\
\hline Pharma & 0.73 & 0.68 & 0.72 & 0.76 & 0.77 & 0.85 & 0.87 & 0.86 & 0.82 \\
\hline Housing & 0.72 & 0.68 & 0.70 & 0.75 & 0.77 & 0.81 & 0.84 & 0.82 & 0.86 \\
\hline Power & 0.59 & 0.50 & 0.52 & 0.64 & 0.69 & 0.73 & 0.83 & 0.64 & 0.82 \\
\hline Transport & 0.68 & 0.59 & 0.67 & 0.74 & 0.77 & 0.78 & 0.85 & 0.79 & 0.74 \\
\hline FMCG & 0.46 & 0.47 & 0.55 & 0.68 & 0.67 & 0.69 & 0.81 & 0.57 & 0.72 \\
\hline Agriculture & 0.63 & 0.57 & 0.60 & 0.67 & 0.71 & 0.76 & 0.89 & 0.76 & 0.79 \\
\hline$C D$ & 0.55 & 0.43 & 0.52 & 0.64 & 0.65 & 0.75 & 0.86 & 0.72 & 0.86 \\
\hline Diversified & 0.78 & 1.20 & 0.76 & 0.82 & 0.81 & 0.86 & 0.96 & 0.93 & 0.89 \\
\hline Overall & 0.96 & 0.50 & 0.53 & 0.64 & 0.69 & 0.73 & 0.84 & 0.64 & 0.82 \\
\hline
\end{tabular}

underlying stocks is better captured for 64 days period. The results also suggest that CAPM will be supported more if one can use returns that are measured during this period. An even more interesting result appears from observing the CAPM beta on un-decomposed returns and the average beta for wavelet scales. A closer glimpse particularly on Mid and Small Cap betas shows that the CAPM beta is essentially an average of wavelet betas which means that but the later provides a resolution more appropriate and hence need to be considered in a realistic risk assessment of securities. These results are the unique contribution of this study.

\section{Conclusion}

Under the homogenous expectations assumption, the CAPM model fails to recognize diverse kinds of market participants seeking investments for different time horizons. For instance, there are traders who take a very long view for the investment and consequently concentrate on what is termed as 'market fundamentals'. These traders ignore ephemeral phenomena. In contrast, other traders trade on a much shorter time-scale and as such are interested in temporary deviations of the market. And yet other traders may operate in the market for which even a day is long time. Each of these classes of traders analyzes their own trading with their own perception of yardstick, consistent with their trading horizons. However, econometric tools were not adequate to characterize and break down the complex heterogonous market behavior. With this background, this paper provides a comprehensive insight about beta risk measurement by resorting to wavelet analysis as it allows one to evaluate simultaneous time-frequency varying features of the given return series within unified framework. Based on the empirical findings, it is argued that the conventional beta estimate based on CAPM is near to the "average" of the wavelet beta estimates. The results indicate that the traditional ways of estimating beta through picking an arbitrary time scale to measure return and simply run CAPM model to estimate beta may not be statistically appropriate since a lot of information about beta dynamics across different intervals could be lost in terms of reduced observations. The results further suggest that the 
market risk for stocks of large capitalized companies are more stable than mid and small capitalized stocks. This implies that large cap investors are more tolerant with the fluctuations in the stock prices or changes in the business cycles of their companies. The results provide the importance of capturing the variation in beta through time in predicting market return for small and mid cap investors. The paper leaves a huge scope to consider return aspect to conduct a thorough investigation of capital asset pricing model across simultaneous time frequency domains.

\section{Endnotes}

${ }^{1}$ As Percival and Walden (2000) note, the MODWT is also commonly referred to by various names in the wavelet literature. Equivalent labels for this transform are non-decimated DWT, time-invariant DWT, un-decimated DWT, translation-invariant DWT and stationary DWT.

${ }^{2}$ Ramsey, J. (2002). Wavelets in economics and finance: Past and future. Studies in Nonlinear Dynamics and Econometrics 6:1-27.

${ }^{3}$ Out of BSE-500, the required data was available for only 311 companies. Selected companies were classified into their respective industries.

${ }^{4}$ The data source for individual stocks and the BSE- 500 index were retrieved from CMIE Database Prowess. The MIBOR daily rates were collected from NSE official website.

\section{Acknowledgements}

Authors are grateful to Wayne King Senior Software Engineer Signal and Array Processing Math works USA for his valuable comments.

\section{Funding}

The expenditure incurred for data collection and subscription of software's was adjusted with the University Grants Fellowship received during our PhD Tenure.

Availability of data and materials

The dataset used/analyzed during the current study are available from the corresponding author on reasonable request.

\section{Authors' contributions}

AS carried out the data compilation and estimation process for derived coefficients using Matlab and R package. He is also responsible for the corresponding interpretation of coefficients. AMT participated in the drafting of manuscript including sequence alignments. QF is responsible for the review section and statistical analysis. All authors read and approved the final manuscript.

\section{Ethics approval and consent to participate}

We declare that the manuscript follows all ethical standards of the Journal of Financial Innovation.

\section{Consent for publication}

All the authors mutually agreed to publish this research in Journal of Financial Innovation.

\section{Competing interests}

The authors declare that they have no competing interests.

\section{Publisher's Note}

Springer Nature remains neutral with regard to jurisdictional claims in published maps and institutional affiliations.

Author details

${ }^{1}$ Indian Institute of Technology Madras, Chennai, India. ${ }^{2}$ Department of Statistics, Pondicherry University, Kalapet, India.

Received: 5 October 2017 Accepted: 1 August 2018

Published online: 31 August 2018

\section{References}

Baesel JB (1974) On the assessment of risk: some further considerations. J Financ Econ 29:1491-1494

Bart J, Masse IJ (1981) Divergence of opinion and risk. J Financ Econ 16:23-34 
Bekaert G, Harvey CR (1997) Emerging equity market volatility. Journal of Financial Economics. 43:29-77

Black F (1972) Capital market equilibrium with restricted borrowing. J Financ Econ 45:444-454 24

Blume ME (1971) On the assessment of risk. J Financ Econ 26:1-10

Deo M, Shah A (2012) Scaling properties of systematic risk: a new evidence from wavelet analysis. J Financ Econ 8:283-289

Fama EF (1965) The behavior of stock-market prices. J Financ Econ 38:34-105

Fama EF, French KR (2004) The capital asset pricing model: theory and evidence. J Financ Econ 18:25-46

Fernandez V (2006) The CAPM and value at risk at different time-scales. J Financ Econ 15:203-219

Gençay R, Whitcher B, Selçuk F (2005) Multi-scale systematic risk. J Financ Econ 24:55-70

Gordon AJ, Norman CN (1980) On the estimation and stability of beta. J Financ Econ 15:123-137

Handa P, Kothari SP, Wasley C (1989) The relation between the return interval and betas: implications for the size effect.

J Financ Econ 23:79-100

Harvey R (1989) Time-varying conditional covariance's in tests of asset pricing models. J Financ Econ 24:289-317

Hiroshi Y (2005) Wavelet-based beta estimation and Japanese industrial stock prices. J Financ Econ 12:85-88

Kalman RE (1960) A new approach to linear filtering and prediction problems. J Financ Econ 82:35-45

Keynes JM (1930) A treatise on money: the pure theory of money. Cambridge University Press

Levhari D, Levy H (1977) The capital asset pricing model and the investment horizon. J Financ Econ 59:92-104

Levy M, Levy H (1996) The danger of assuming homogeneous expectations. J Financ Econ 52:65-70

Markowitz HM (1952) Portfolio selection. J Financ 7:77-91

Markowitz HM (1959) Portfolio selection. John Wiley and Sons, Inc, New York

Miller E (1977) Risk, un-certainty, and divergence of opinion. J Financ Econ 32:1151-1168

Moonis SA, Shah A (2003) Testing for time-variation in beta in India. J Financ Econ 2:163-180

Muller U, Dacorogna M, Davé R, Olsen R, Pictet O, Weizsacker JV (1997) Volatilities of different time resolutions -

analyzing the dynamics of market components. J Financ Econ 24:213-239

Oral E, Gazanfar U (2017a) Dynamic correlation of Eastern and Western markets and forecasting: scale-by-scale waveletbased approach. J Financ Econ 4:1-22

Oral E, Gazanfar U (2017b) Co-movement of precious metals and forecasting using scale by scale wavelet transform. J Financ Econ 4:1-21

Percival D, Walden A (2000) Wavelet Methods for Time Series Analysis, Cambridge Press Cambridge.

Ramsey J (2002) Wavelets in economics and finance: Past and future. Studies in Nonlinear Dynamics and Econometrics, $6: 1-27$.

Rua A, Nunes LC (2012) A wavelet-based assessment of market risk: the emerging markets case. J Financ Econ 52:84-92

Scholes M, Williams J (1977) Estimating betas from non-synchronous data. J Financ Econ 5(25):309-327

Scott E, Brown S (1980) Biased estimators and unstable betas. J Financ Econ 35:49-55

Sharpe WF (1964) Capital asset prices: a theory of market equilibrium under conditions of risk. J Financ Econ 19:425-442

\section{Submit your manuscript to a SpringerOpen ${ }^{\circ}$ journal and benefit from:}

- Convenient online submission

- Rigorous peer review

- Open access: articles freely available online

- High visibility within the field

- Retaining the copyright to your article

Submit your next manuscript at $\boldsymbol{\nabla}$ springeropen.com 